\begin{tabular}{c} 
journal homepage: http://ijiemjournal.uns.ac.rs/ \\
International Journal of Industrial \\
Engineering and Management \\
volume $12 /$ No $2 /$ June $2021 / 129-140$ \\
\hline
\end{tabular}

Original research article

\title{
Digital twin testbed and practical applications in production logistics with real-time location data
}

\author{
J. Baalsrud Hauge ${ }^{\mathrm{a}, \mathrm{b}}$, M. Zafarzadeh ${ }^{\mathrm{a}}$, Y. Jeong ${ }^{\mathrm{a} *}$, Y. Lic, W. Ali Khilji ${ }^{\mathrm{a}}$, C. Larsen ${ }^{\mathrm{d}}$, M. Wiktorsson ${ }^{\mathrm{a}}$ \\ ${ }^{a}$ KTH Royal Institute of Technology, Södertälje, Sweden; \\ $b^{b}$ Bremer Insitut fur Produktion und Logistik GmbH (BIBA), Bremen, Germany; \\ ${ }^{C}$ Fraunhofer-Chalmers Centre for Industrial Mathematics, Gothenburg, Sweden; \\ ${ }^{d}$ Virtual Manufacturing, Gothenburg, Sweden
}

\section{A B STRACT}

Nowadays, digital twins exist everywhere in various fields. However, an analysis of existing applications in manufacturing and logistics revealed that many entirely apply the concept. To identify when a complete implementation of the concept is beneficial, we analyse the need and the implications within production logistics. This study also presents an architecture supporting integrating a digital twin into production logistics and a corresponding application scenario. Based on this, we have derived practical applications. Each application is applied to different situations, and actual benefits can overcome the limitations of the previous studies.

\section{ARTICLE INFO}

Article history:

Received January 16, 2021

Revised May 13, 2021

Accepted May 14, 2021

Published online May 26, 2021

Keywords:

Automated guided vehicle;

Digital twin;

Production logistics;

Simulation;

Real-time location

"Corresponding author:

Yongkuk Jeong

yongkuk@kth.se

\section{Introduction}

To reduce the often high costs of logistics operations with low added-value, [1], [2] stakeholders try to implement technologies to offer the same or better services at a lower cost [3]-[5]. But the implementation of technology in the existing environment faces some challenges [6], [7]. A key challenge is assessing how different new components will interact with the existing systems such as a typically challenge when dealing with technology introduction in complex systems [5]. The usage of safe environments like testbeds or pure simulation environments can, with some limitations, contribute to overcoming these challenges [8]. Such a limitation is related to the decentralised decision-making process carried out by the autonomous robots (like automated guided vehicles (AGVs) or intelligent cargo) [1], [3], [9], which is hard to simulate since we know little about the used algorithm. The usage of digital twins can help overcome this challenge since it can visualise the differences between the optimal path based on algorithms and the optimal route based on the inbuilt decision-system in the AGV.

The focus of this article is to discuss how digital twins can contribute to support the decision-making 
process of selecting the right components for a specific task, both related to the degree of automation and the digitalisation of the operations. The rest of the article first describes the requirements on a digital twin used for decision-making; secondly, how manipulating the digital twin can understand the system and components interactions better before the testbed we are using is described in more detail. Finally, we describe how the different components are integrated and interacting and the first results. The last section discusses the main challenges and the next steps. The following research questions are designed to meet the goal of this paper:

(RQ1) When do we need digital twins and real-time location data in production logistics?

(RQ2) What kind of practical applications of digital twins and real-time location data can benefit production logistics operation?

(RQ3) What is the actual benefit of having digital twins instead of digital shadow in production logistics?

\section{Related works}

\subsection{Technology integration challenges}

Technology introduction has a significant impact on operational processes both at the managerial and operative levels [9]. Therefore, it is not sufficient to only pay attention to the technical aspects and predict their outcomes and, based on that, make the right decisions [10], [11]. According to Yao et al. [12], digital twins can support the planning and prediction of the working processes.

The term digital twin (DT) referred back to 2002 and referred to a component concept- a virtual and a physical component, and the bi-directional information exchange in between [13]. The uptake in several research domains, including production, came around ten years later [14]-[16], and a similar concept is known from NASA's concepts for space shuttles [17].

Today, DT is a widely used concept; however, several different definitions exist [14], [18]-[22]. Furthermore, applications named DTs also show a wide variance in terms of functionalities. The original meaning Grieves [13] had back in 2002 comprised a bi-directional information flow and a physical and a virtual component. Still, in the literature, several DT examples exist with only an information exchange from the physical to the virtual component but not vice versa. For this type, the term digital shadow (DS) is also in use [23]. In addition to several definitions, some works extend the Grieves original concept, i.e., Tao and Zhang [24] extend Grieves original system with two other concept components: services and data as explicit.

Regarding the definition of a DT, in this work, we build upon the definition by Tao et al. [25] as well as on the characteristics of a DT as described in [9] in respect to a) real-time reflection b) interaction and convergence and c) self-evolution. As described in the introduction, the main challenge of introducing concepts like Internet-of-Things (IoT), cyber-physical system (CPS), and Industrie4.0, etc., in existing environments is assessing how the technology will affect the whole organisation and interact with existing solutions. According to [26]-[28], DTs can be manipulated to support the decision-making process. Regarding the assessment of autonomous and decentralized decision-making, which is required in CPS [29], [30], it is hard to model these with the proper interactions with other system components and with the correct routing (decided upon by the autonomous object) [3], [12]. However, based on experiences on importing sensor data from a lab environment into the virtual environment, comparing the routes and interaction with the first simulations, and then modify and manipulate these data in the DT (by using game mechanics), a better system understanding can be achieved [31].

\subsection{Digital twins in production and logistics}

Out of the five definitions referred to in the introduction, three are from the manufacturing application domain [19]-[21]. Jones et al. [32] claim that a reason for the inconsistency is that the scientific description is still incomplete. The following paragraphs will introduce different aspects of DTs' maturities and functionalities, focusing on DT in the production and logistics area. It is based on a comprehensive literature review (own work in progress) distinguishing more than ten different application domains for DTs. While they found 17 contributions in the field of manufacturing [23], [25], [32]-[43], only one Lim et al. [44] is in logistics, while automotive, aerospace also in many cases could be considered to be manufacturing. A more detailed look into the identified articles sheds some more light on understanding how DT is used. Tao et al. [25] present an overview of how DTs are used in different parts of the product life cycle. Specifically relevant for our work is here the observations on DT in the production, illustrating how DTs can contribute to making a production 
process more reliable, flexible, and predictable.

Furthermore, in their review, Negri et al. [33] state that their analysis has shown that the understanding of DT is diverging and that the research is in its infancy. But it also motivates why this concept can be beneficial to the sector, since it allows the representation to reflect the current status of the system and to perform real-time optimizations, decision making and predictive maintenance according to the sensed conditions have identified that within the field of manufacturing [40, p946]. In their literature review, Lim et al. [44, p12] describe a set of examples of how DT is used within logistics- in the field of Hunan-Robot collaboration, warehouse management, and decision-making support. The analysis of the current state of the art of DT applications in production and logistics shows progress in the maturity of the application from DS to fully DT, but that there are still challenges to overcome specifically in logistics with all its interactions and movements. Thus, DT applications in manufacturing seem currently more mature than the examples within logistics.

The following sections will connect to the examples given in Lim et al. [37] for route optimization in warehouses or shop floors and explain how simulations can be used. This is illustrated in a case study illustrating how the usage of known algorithms applied in the virtual context can help overcome the lack of access to the algorithms of AGVs and how the combination can improve the overall system performance.

\subsection{Route planning in production logistics}

To solve the route planning (also called motion planning or path planning in the field of robotics) problem, sample-based planning methods such as probabilistic roadmap (PRM) [44] and rapidly-exploring random tree (RRT) [45] can be used to compute a collision-free path for a manikin or an AGV by connecting independently and identically distributed random points in the configuration space. In this work, we assume that both manikin and AGV can translate (on a 2D plane) and rotate (around the plane normal). Hence, their configurations can be represented using three parameters $(x, y, \theta)$, where $\theta$ represents orientation. Even though sample-based planning methods are fast, they can produce unusual-looking paths. Furthermore, a sample-based planning method cannot guarantee to create the same path every time it is executed because these methods rely on random sampling (i.e., they are not deterministic). Instead, we choose a search-based planning method (SBPL [46]). Planning with search-based methods consists of two parts: representing the problem as a graph (i.e., discretize all possible states, more specifically the $\mathrm{x}, \mathrm{y}$, and orientation in this work), solving the graph with graph-search methods. A path returned by a graph-search method such as anytime repairing A* (ARA*) [47] is simply a series of adjacent states in a graph. Suppose the AGV is car-like (i.e., it is a nonholonomic vehicle: it has only two controls, but its configuration space has dimension 3). In that case, two adjacent states may not reflect the kinodynamic constraints of the AGV. To solve this problem, we define two sets of motion primitives: the first set contains pre-computed motions that the manikin can take, whereas the second set includes pre-computed motions that the AGV can handle. There is also a cost associated with each motion. Since we do not want to reverse the AGV frequently, a high cost might be assigned to the corresponding motion primitive. By superimposing these motion primitives on the graph, an adjacent state represents what the manikin and AGV can smoothly transition to, even though it is not necessarily spatially adjacent.

\subsection{Simulation in production logistics}

Mardberg [48] introduced a novel digital factory layout tool that optimizes the layout of machines while taking into account human factors and ergonomics. This model contains the following components: the properties of the devices, their mutual relationships, the distances travelled by workers or AGVs, and other constraints (e.g., the placements of electric outlets). In this work, they applied this digital layout tool to optimize the layout of a fulfilment centre (i.e., to determine the optimal placements of the storage shelves and in which bin each part should be stored).

\section{Methodology}

In this paper, a case study was selected as the primary method to perform this research. The case study method facilitated an in-depth understanding of the potentials benefits and challenges of employing real-time data production logistics [49]. According to Meredith [50], a case study can be considered as an appropriate method when the amount of unknown knowledge is considerable. A physical testbed was developed to prepare the situation to perform experiments to evaluate production logistics digitalisation scenarios. A DT of the physical environment was developed to facilitate the simulation of complex scenarios. First, the track and trace of an AGV are 
studied to reveal the potential benefits of using DT in a production logistics environment. In addition, the process of route planning simulated through IPS simulation software aiming to find the optimal route. The physical environment dimensions and coordinates are transferred to IPS representing the natural environment. An AGV model capable of manoeuvring around similar to the AGV movements in the physical lab was imported to the model. Second, the kitting process was studied to evaluate real-time data and DT use to streamline the working process. Third, real-time forklift tracking is studied within an industrial environment to examine the operation's DT.

\section{Digital twin testbed and applica- tions for production logistics}

As described in the introduction and the related work section, a DT can mirror or simulate complex scenarios very close to reality [9], [30], [31], [51]. The DT provides an opportunity to be used as a learning and awareness-increasing tool in which operators or management can use virtual reality (VR) tools to experience the natural working conditions with high precision [52]. It can also support introducing new technologies and installations within the existing physical environment [52], [53]. Furthermore, the examples are given above also indicate how simulation can be used to improve the efficiency of a logistics process by supplying the workers with the necessary information. In this case, we simulate the working processes and observe the material movements, machines, robots, etc., and their interactions [54]. I.e., in these cases, we need both a visualisation that allows the employees either working in the warehouse or making a decision related to technical support on one hand side (requires a shadow). Still, we might also need to be able to interact with the entire operations in the physical component based on what we observe virtual (requires a twin). In the following case study, we have set up a set of experiments to investigate better when we need real-time information in a bidirectional way and sufficient with a one-way integration and the requirements in real-time. We have used a testbed that focuses on production logistics, i.e., it focuses on the material and information handling within the production system, with solid and integrated interfaces to supply chain management. This physical testbed should allow testing of a) man to material technologies, b) robot to material, c) material to man, and d) material to robot. Furthermore, to realise the latter, it should explore the usage of a real-time locating system (RTLS) and the interaction and operation of different AGVs. [55], [56]. Based on the analysis of existing applications, we here specifically look at the impact of b) robot to material technology support, real-time information, interoperability, decentralized decisions core technology areas [51].

\subsection{Components and architecture}

To evaluate the applications in production logistics and address RQ2, the lab environment and the lab components, including the AGV, carts, and parts, are simulated in a 3D environment using Unity. Real-time tracking of the AGV in the model realised

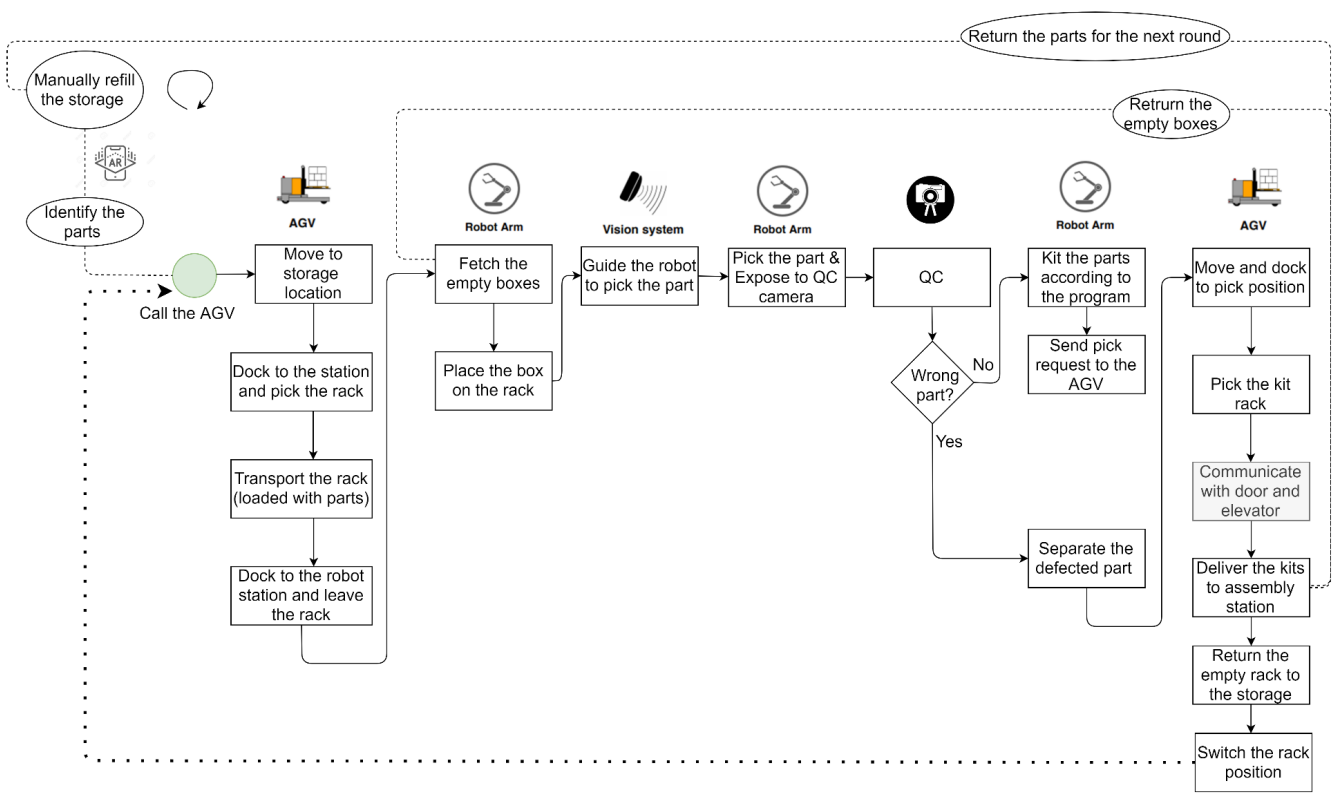

Figure 1. Material flow process within the testbed 
through integrating the physical lab and the DT through IoT technology. Also, data from the physical environment transfers to the DT, and this system is controlled by Node-Red application. As shown in Figure 1, the focus of the first setup is on connecting equipment and RTLS since these are a prerequisite for realising a transparent and efficient material flow from one warehouse to a production site.

The initial demonstration scenario is to kit three different parts in two other kits by introducing a collaborative robot, which is known as UR, an AGV, an intelligent vision camera, and an RTLS. The AGV transport the parts from the storage position to the UR robot station, which performs the kiting with the help of the vision system. The AGV will transport the kitted parts to the assembly station at the end. The physical objects and the DT are integrated into a single system to investigate the problems mentioned in the introduction, which components communicate via a data streaming bus. Figure 2. represents the system architecture for the production logistics DT. This system interconnects all components to make use of the DT of the real-world scenario. As a first step, communication between each component was realised.

A data streaming bus, which will hold and serve messages wherever needed, was chosen for this purpose. Apache Kafka is the data streaming platform selected for this purpose. Implementing the data streaming bus facilitated the data exchange among data producers and data consumers regardless of their data format and integration issues. Data can be consumed in real-time as well as be stored for later analysis. Secondly, there is an application layer, which is required to write business logic or calculations. Node-Red application is responsible for integrating and controlling the business logic and process steps. In addition, data storage and DT ought to be connected through the application layer. Since the components need to be visualised to support the decision-making, the Unity application is used to visualise the models and show the behaviour of the physical components in real-time. AGV controller and robot controller are two features developed to control the process via a graphical interface. The RTLS system is connected to the cloud and has interaction with the application layer and data streaming bus. The IPS simulator was used to find the optimal route for the AGV. To complete the integration, message queuing telemetry transport (MQTT) bridged the connection to the Node-Red application.

In the digital environment, replica components of the physical environment are defined. Each replicated component has to go through the data streaming bus and application layer to send and receive information from the physical environment since the operating systems, data formats, and communication methods are different by each component.

Next describes communication between the components in more detail: a) The AGV communicates via web API to share location data and various parameters such as battery status, speed, and orientation of the AGV. b) The collaborative robot communicates over a transmission control protocol (TCP) socket connection to share different data types such as trajectories. Here, the robot is treated as a server and connected via a TCP client application. c) The RTLS system uses API to retrieve location data of

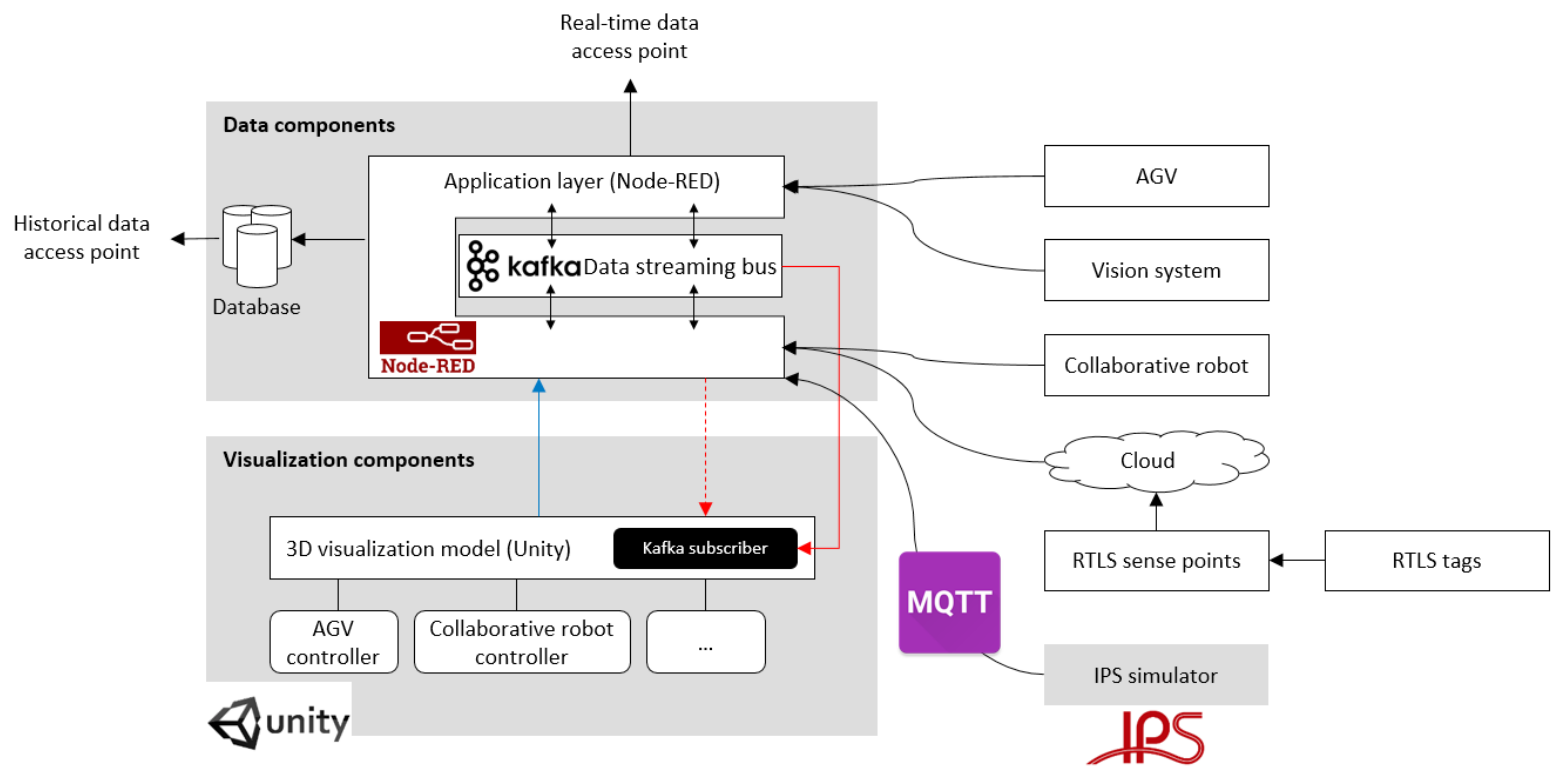

Figure 2. Production logistics digital twin system architecture 
these various objects. d) IFM 3D sensors used as vision systems and connected via Ethernet to NodeRed. The data sent to a middleware that translates them into the collaborative robot coordinates. In this case, the primary function of the vision system is robot guidance to kit the parts according to the scenario shown in Figure 1. e) To visualise the DT, Unity is used along with some complex prefabs like AGV and collaborative robot, delivered by the supplier or modelled using IPS. Each controller is responsible for fetching data or controlling the machine. f) Data storage is used for the long-term data solution for data analytics or data mining to replay a particular scenario. The following section describes the different possibilities we have for the models in the DT and outlines what we currently look at.

The digital environment mirrors the physical environment in the testbed and allows 'manipulation' to allow experimentation with different technologies and equipment before the decision in a virtual world.

\section{Applications for logistics operations analysis}

\subsection{Real-time tracking of AGV}

The DT is used both with pure simulation data and with data from what we already have in operation to figure out the integration and interaction of $\mathrm{AGV}$ and picking robot investigating how the AGV is doing its mission scheduling and how this can be improved. Interaction and interference with existing systems and deciding upon the suitable new object (like the type of AGV) is a challenge, as described earlier. Secondly, another challenge is that there is limited information about the algorithm the AGVs are using for calculating their path. However, we have full control over the algorithm, the DT of the AGV (modelled using IPS), which is the optimised algo- rithm. Comparing the results of the DT optimised algorithm and the physical movements of the AGV can give valuable information on the behaviour and thus help in the assessment of the suitability for implementation in different environments. Furthermore, the integration of the physical data in the DT allows a better visualisation that can be used for: a) Detailed investigation of the exact movements of the AGV or collaborative robot (as shown in Figure 3). b) Using a replay function and combining different experiments in one single DT scenario helps understand the variation in movements and recognise patterns (i.e., continuously occurring deviations between real movements and optimised calculated paths). c) To explore an environment, which might have no or limited access (either using VR, AR, or a 3D computer model on the PC). This can be used for teaching purposes to explain the interaction of the different components and let the students try out simple actions in a safe environment. Figure 3 illustrates this possibility. The picture to the left shows the digital model of the lab environment. The viewer can use a camera to move around and experience the room. Besides the detail of the models- here, the path of the $\mathrm{AGV}$ - is visualised. The image to the right shows the physical AGV (data imported in the Unity model via the communication bus).

The DT can start the different physical operations via the DT interface. This opens up the possibility for carrying out experiments in risk areas. In this case, except few individuals, most of the personnel do not have convenient access to operate the physical equipment on-site, can perform experiments through DT. This increases the possibilities to utilise expensive lab equipment to a higher degree.

\subsubsection{Optimal route planning in a dynamic environment}

Most of the AGVs in the market has vision sen-
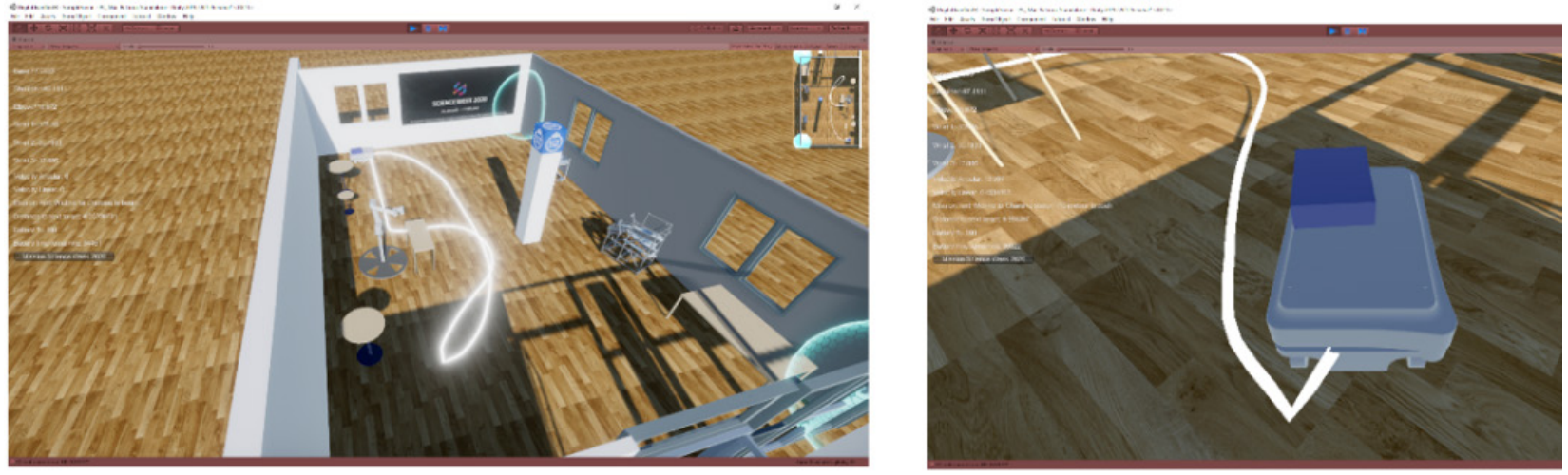

Figure 3. Tracking AGV in real-time within the digital twin 
sors such as camera and laser. This allows AGVs to scan their surroundings and navigate the paths that avoid obstacles. AGV receives missions with a fixed destination in the production logistics environment and calculates the optimal route using the AGV's route searching algorithm. Suppose an obstacle is suddenly appeared on the path while moving as planned initially. In that case, AGV pauses the operation, and the new surroundings are rescanned to update the optimal way, as shown in Figure 4 (a). In this case, the AGV can avoid obstacles, but it has the disadvantage that it cannot dynamically respond to barriers that were not found when initially planned. Also, the operation must be paused while recalculating the new path.
This problem can be solved by utilizing the DT proposed in this study. The DT, which collects real-time data from the production logistics environment, can update the surrounding environment periodically. Therefore, the AGV can respond to unexpected obstacles during the operation that were not found when it calculates the optimal path at the beginning of its delivery task. Unlike the previous method, which is without a DT, obstacles can be avoided even in a dynamic environment because the surroundings are scanned. The traveling path is updated accordingly, as shown in Figure 4 (b).

However, the DT cannot calculate the optimal path on its own. Therefore, it periodically checks the criteria defined in advance in DT and calls the IPS
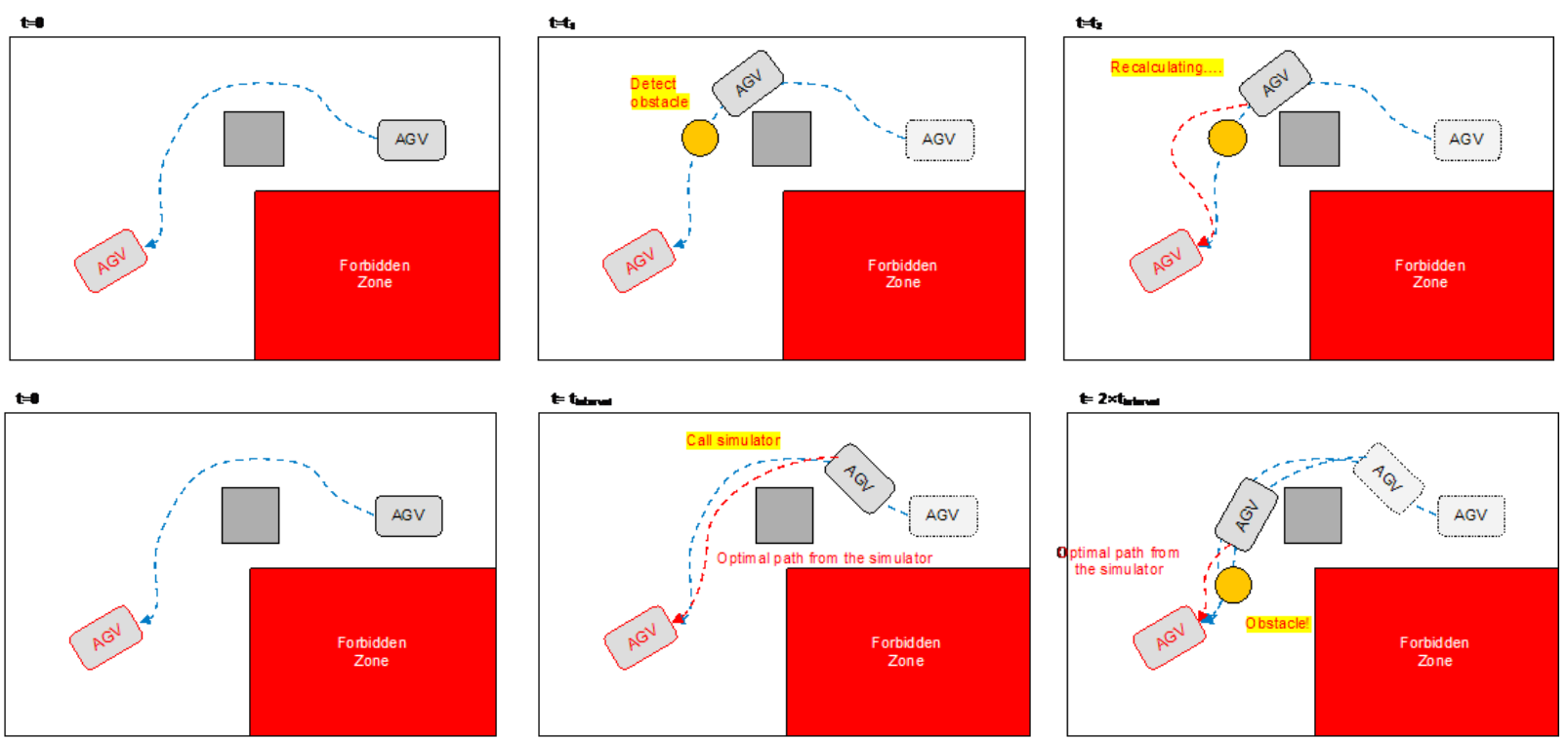

Figure 4. Optimal route planning without digital twin

( $a$-three diagrams at the top) and with digital twin ( $b$ - three diagrams at the bottom)

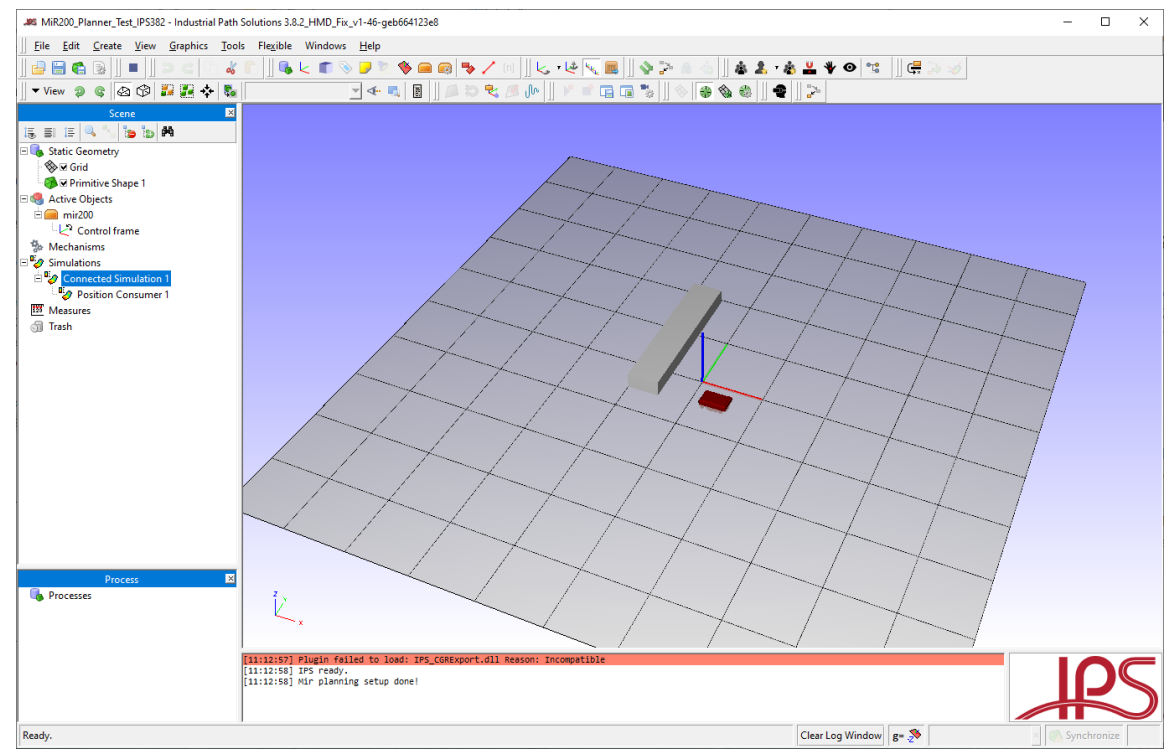

Figure 5. Optimal route planning simulator and AGV 
simulator to calculate the optimal route when a new obstacle is detected, as shown in Figure 5.

The IPS simulator contains an algorithm that derives the optimal path for a given starting point, destination, and obstacles. The simulator validates the route from the algorithm, and if valid, the updated course is transferred with a list of waypoints to the AGV. Figure 6. shows such a process, and based on this, we tried to perform various experiments by scenarios in the production logistics lab. However, the actual investigation could not be completed due to the limited physical access restrictions.

\subsection{Kitting}

Using DS can facilitate the study of kitting process in production logistics. Analysing the collaboration between AGV, UR robot, and vision system is one of the use cases. Different setups can be examined within a DS, which might be too costly or difficult to implement in a natural environment. Besides, it will be possible to add more components to the DS, such as several $\mathrm{AGVs}$ to analyse the natural environment's behavior. Physical constraints and collisions can also be studied by using a DS.

To perform the kitting process, UR10 collaborative robot equipped with a vacuum gripper in collaboration with AGV Mir200 was used. Ten parts are placed on a cart and exposed to the vision system. Four different types of pieces are placed in two kits

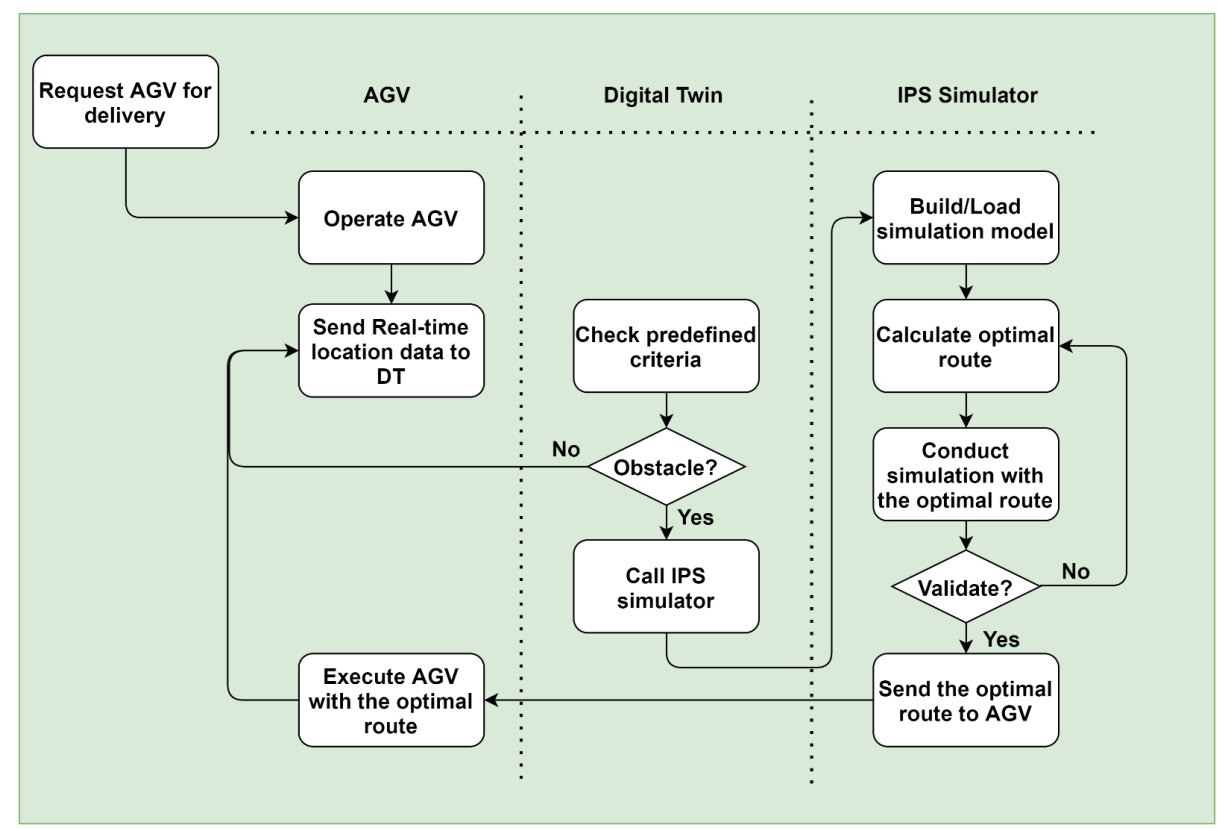

Figure 6. The optimal route planning process with a digital twin in a dynamic environment

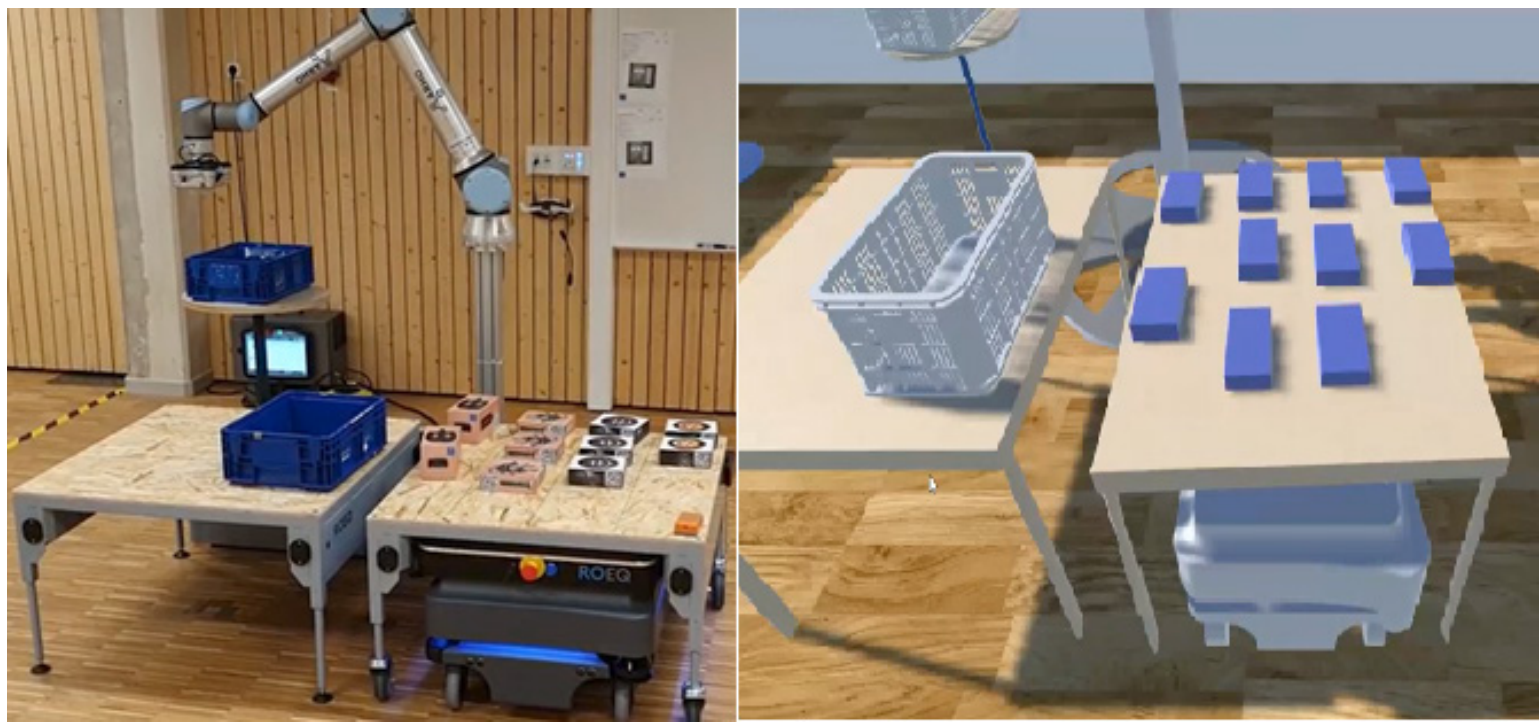

Figure 7. Kitting process in physical and digital environments 
on the next cart. The lab environment and the corresponding DT are shown in Figure 7.

The vision system captures the parts on the cart, and based on the kitting instruction, the UR robot picks the parts and places them on the intended kiting box. Captured picture from the vision sensor translated to coordinates for the UR robot. Figure 8. shows the vision sensor view of the carts. The parts are placed on the cart based on their type, as each row is dedicated to one specific type of part. This design represents a setup close to reality where parts are presented in pallets to picker for kitting. Kitting instruction is communicated via Node-Red, and once the kitting is finished, the $\mathrm{AGV}$ docks to the kit rack and transports the kits to two assembly stations as stated in the scenario.

\subsection{Indoor real-time forklift tracking and operation analysis}

The most significant difference between the DT and digital model or DS is that the results derived from the digital environment are fed back to the physical environment. Even in conventional production logistics environments, decision support using simulation models has been performed a lot. Still, it was hard to find a case of monitoring data in real-time and providing feedback in real-time based on analysing the historical data. We installed the real-time location sensors on the forklifts operating in the production line and collected data in real-time. The collected data includes a unique identification number, timestamp, X, Y, and GPS coordinates, and zone information, as shown in Table 1.

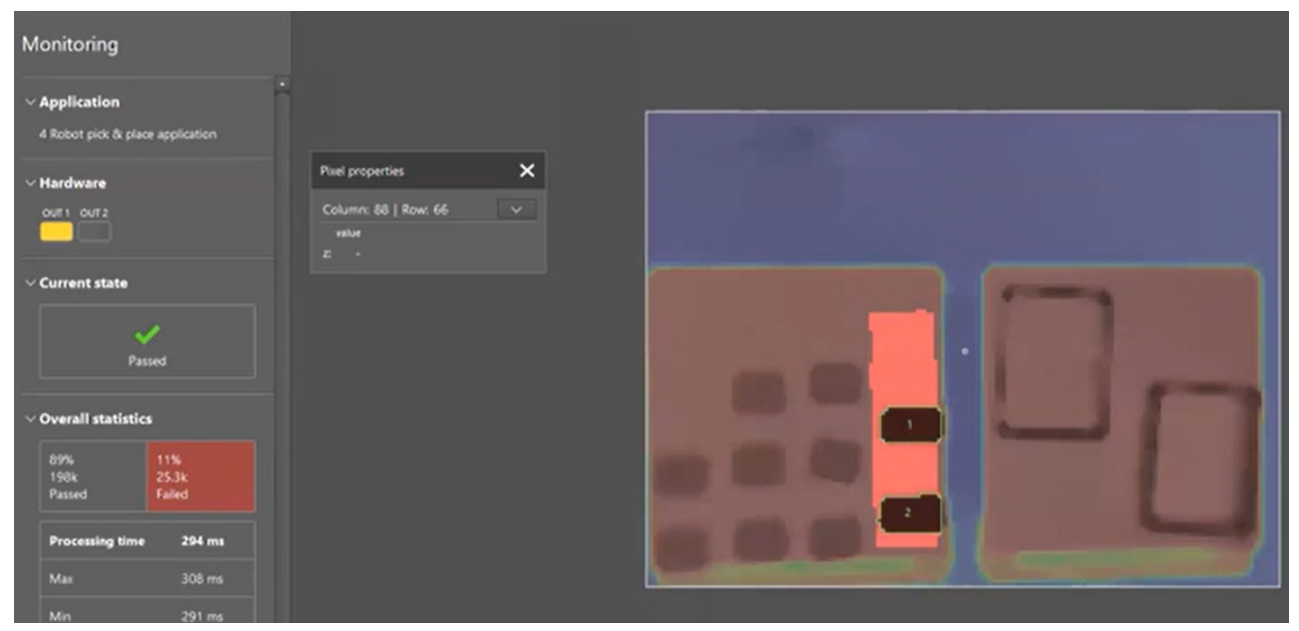

Figure 8. Kitting process in physical and digital environments

Table 1. Raw data set from the real-time location sensors

\begin{tabular}{ccccccccc}
\hline Index & TagID & Timestamp & $\mathbf{X}$ & $\mathbf{Y}$ & Zone & ZoneName & GPS.X & GPS.Y \\
\hline 0 & 00000001 & $2020-12-0707: 33: 34$ & 258 & 19559 & 0 & Transfer & 17,63787 & 59,17988 \\
\hline 1 & 00000001 & $2020-12-0707: 33: 36$ & 258 & 19059 & 0 & Transfer & 17,6379 & 59,17992 \\
\hline 2 & 00000001 & $2020-12-0707: 33: 39$ & 257 & 18562 & 0 & Transfer & 17,63793 & 59,17996 \\
\hline 3 & 00000001 & $2020-12-0707: 33: 41$ & 257 & 18064 & 0 & Transfer & 17,63796 & 59,18001 \\
\hline 4 & 00000001 & $2020-12-0707: 33: 42$ & 257 & 17766 & 0 & Transfer & 17,63798 & 59,18003 \\
\hline 5 & 00000001 & $2020-12-0707: 33: 45$ & 256 & 17267 & 0 & Transfer & 17,63801 & 59,18007 \\
\hline 6 & 00000001 & $2020-12-0707: 33: 46$ & 256 & 16968 & 0 & Transfer & 17,63802 & 59,1801 \\
\hline 7 & 00000001 & $2020-12-0707: 33: 49$ & 256 & 16468 & 0 & Transfer & 17,63805 & 59,18014 \\
\hline 8 & 00000001 & $2020-12-0707: 33: 50$ & 255 & 16170 & 0 & Transfer & 17,63807 & 59,18017 \\
\hline 9 & 00000001 & $2020-12-0707: 33: 53$ & 355 & 15086 & 0 & Transfer & 17,63811 & 59,18026 \\
\hline 10 & 00000001 & $2020-12-0707: 33: 54$ & 361 & 15086 & 0 & Transfer & 17,63811 & 59,18026 \\
\hline 11 & 00000001 & $2020-12-0707: 33: 57$ & 292 & 14876 & 0 & Transfer & 17,63814 & 59,18028 \\
\hline 12 & 00000001 & $2020-12-0707: 33: 59$ & 297 & 14377 & 0 & Transfer & 17,63816 & 59,18032 \\
\hline 13 & 00000001 & $2020-12-0707: 34: 01$ & 301 & 13877 & 0 & Transfer & 17,63819 & 59,18036 \\
\hline 14 & 00000001 & $2020-12-0707: 34: 03$ & 306 & 13377 & 0 & Transfer & 17,63822 & 59,18041 \\
\hline 15 & 00000001 & $2020-12-0707: 34: 05$ & 310 & 12877 & 0 & Transfer & 17,63825 & 59,18045 \\
\hline 16 & 00000001 & $2020-12-0707: 34: 06$ & 313 & 12582 & 0 & Transfer & 17,63826 & 59,18047 \\
\hline
\end{tabular}


In addition, when operating multiple forklifts, we can monitor the condition of each forklift or the distance between the forklifts to prepare for unexpected situations and send a warning alarm to the operator or the physical environment. If the movement pattern of the forklift is different from the historical pattern, it can respond by feedback negative signals. The historical data stored in the server can be used to analyse operational patterns through extensive data analysis or to calculate KPIs such as travel distance and utilization rate. Figure 9. shows this process.

\section{Discussion and Coclusion}

The literature review related to DT in manufacturing and logistics revealed that the research is in its infancy and that there is a significant difference in the maturity of these DT, specifically in the logistics domain. Furthermore, the analysis revealed that quite a few applications are more to be characterised as DSs than DTs. There are many reasons for that: data collection of moving goods or the lack of interaction possibilities with the equipment, as illustrated in operating AGVs. A third reason for having DSs instead of twins may also be that we, in many cases, don't need a fully developed twin. This is the case if we use the mixed reality environment for analysing what happens like required in many planning or training activities- a simple replay function and good visualisation can here be sufficient. At the same time, this would not be sufficient for using it in an operative process or to optimise the behaviour of equipment (here AGV), to which we have no access to change the calculation algorithm. Therefore, the case study shows three different examples on how a DT could contribute to an improved performance of involved equipment on the one-hand side and better opportunities to control and interact on the other hand.

In the next step, it will also be possible to run and manipulate the model from the outside. This will allow us to connect different testbeds to one single digital testbed to look at more considerable challenges. The simulated optimized route in the DT will be compared to the actually chosen path by AGV in the physical environment. Besides, quality control checkpoints need to be located and investigate how to embed this into the material flow process.

\section{Acknowledgements}

This paper is an extended version of the original article published in the 41 st IFIP International Conference on Advances in Production Management Systems (APMS2020) [11].

\section{Funding}

The European Union funded the project on which this paper is based. Central Baltic Interreg program, funding code CB743, and Vinnova Produktion 2030 testbed project Digilog. The responsibility for the content of this publication lies with the authors.

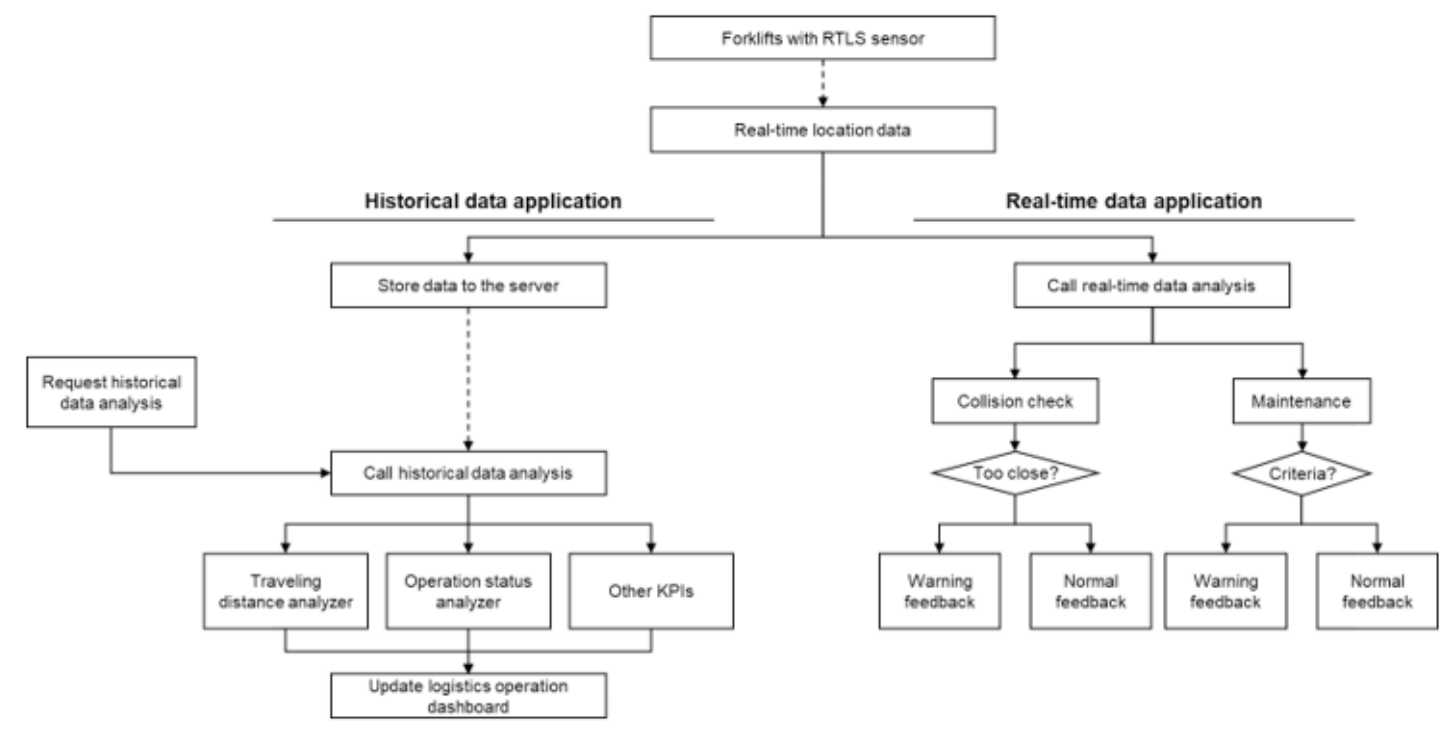

Figure 9. The optimal route planning process with a digital twin in a dynamic environment 


\section{References}

[1] V. Tamás, P. Bányai, and B. Illés, Intelligent Transportation Systems to Support Production Logistics. Cham, Switzerland, 2016.

[2] M. Christopher, Logistics \& supply chain management, 5th ed. Harlow, United Kingdom: Pearson Education, 2016.

[3] M. Forcolin, E. Fracasso, and P. Tumanischvili, Francesco Enterprising, "EURIDICE- IoT applied to Logistcst using the intelligent Cargo concept," in ICE proceedings, 2011.

[4] T. Zunder, H. Westerheim, R. Jorna, and J. T. Pedersen, "Is it Possible to Manage and Plan Co-Modal Freight Transport Without a Centralised System?," Int. J. Appl. Logist., vol. 3, no. 2, 2012.

[5] J. O. Strandhagen et al., "Logistics 4.0 and emerging sustainable business models," Adv. Manuf., vol. 5, no. 359, 2017.

[6] Y. Patil, "6 key IoT Implementation Challenges for Enterprises to consid-er." [Online]. Available: https://www. saviantconsulting.com/blog/iot-implementation-challeng es-enterprises.aspx. [Accessed: 20-Jan-2001].

[7] M. W. I. Ek and P. Frykblom, "Hur kan staten främja användandet av digitaliseringens möjligheter i näringslivet?," Östersund, 2018.

[8] H. Bosson and J. Ingmarsson, "Digitalisering av svensk industri-kartläggning av svenska styrkor och utmaninger," 2016.

[9] F. Tao, J. Cheng, Q. Qi, M. Zhang, H. Zhang, and F. Sui, "Digital twin-driven product design, manufacturing and service with big data," Int. J. Adv. Manuf. Technol., vol. 94, no. 9-12, pp. 3563-3576, 2018.

[10] M. Crnjac, I. Veža, and N. Banduka, "From concept to the introduction of industry 4.0,” Int. J. Ind. Eng. Manag., vol. 8, no. 1, pp. 21-30, 2017.

[11] Hauge, Jannicke Baalsrud, Y. Zafarzadeh, Masoud, Jeong, Y. Li, and M. Khilji, Wajid Ali, Wiktorsson, "Digital and Physical Testbed for Production Logistics Operations," in The Path to Digital Transformation and Innovation of Production Management Systems, Cham: Springer International Publishing, 2020, pp. 625-633.

[12] A. K. F. Yao, M. Ahmad, B. Ahmad, R. Harrison, and A. W. Colombo, "Optimizing the Scheduling of Autonomous Guided Vehicle in a Manufacturing Process," in IEEE 16th International Conference on Industrial Informatics (INDIN), 2018, pp. 264-269.

[13] M. W. Grieves and J. Vickers, "Digital Twin: Mitigating Unpredictable, Undesirable Emergent Behavior in Complex Systems," in Transdisciplinary Perspectives on Complex Systems, Springer International Publishing, 2017.

[14] D. Glaessgen, E. and Stargel, "" The Digital Twin Paradigm for Future NASA and U.S. Air Force Vehicles," in "53rd AIAA/ASME/ASCE/AHS/ASC Structures, Structural Dynamics and Materials Conference, 2012.

[15] E. J. Tuegel, "The Airframe Digital Twin: Some Challenges to Realization," in AIAA/ASME/ASCE/AHS/ASC Structures, Structural Dynamics and Materials Conference, 2012.

[16] M. P. Reifsnider K, "Multiphysics stimulated simulation digital twin methods for fleet management.," in In: 54th AIAA/ASME/ ASCE/ AHS/ASC Structures, Structural Dynamics, and Materials Conference, 2013, p. 1578.

[17] S. M, C. M, and Doyle R et al, "DRAFT modeling, simu- lation, information technology \& processing roadmap.," 2010.

[18] S. Boschert and R. Rosen, Digital twin the simulation aspect. Basel: Springer International Publishing, 2016.

[19] Y. Chen, “'Integrated and Intelligent Manufacturing: Per spectives and Enablers.," Engineering, vol. 3, no. 5, pp. 88-595, 2017.

[20] N. Schleich, B., L. Anwer, Mathieu, and S. Wartzack., "Shaping the Digital Twin for Design and Production Engineering.' -," CIRP Ann. Manuf. Technol., vol. 66, no. 1, pp. 141-144, 2017.

[21] R. Stark, C. Fresemann, and K. Lindow, "Development and operation of Digital Twins for technical systems and services," CIRP Ann. - Manuf. Technol., vol. 68, no. 1, pp. 129-132, 2019.

[22] Y. Lu, C. Liu, K. I. K. Wang, H. Huang, and X. Xu, "Digital Twin-driven smart manufacturing: Connotation, reference model, applications and research issues," Robot. Comput. Integr. Manuf., vol. 61, no. August 2019, p. 101837, 2020.

[23] P. W. Kritzinger, M. Karner, G. Traar, J. Henjes, and W. Sihn, "Digital Twin in manufacturing: A categorical literature review and classification," IFAC-PapersOnLine, vol. 51, no. 11, pp. 1016-1022, 2018.

[24] F. Tao and M. Zhang., "Digital Twin Shop-Floor: A New Shop-Floor Paradigm Towards Smart Manufacturing.", IEEE Access, vol. 5, pp. 20418-2042, 2017.

[25] F. Tao, Q. Qi, A. Liu, and A. Kusiak, "Data-driven smart manufacturing," J. Manuf. Syst., vol. 48, no. January, pp. 157-169, 2018.

[26] B. Brenner and V. Hummel, "Digital twin as enabler for an innovative digital shopfloor management system' in the ESB logistics Learning Factory at Reutlingen University," Procedia Manuf., vol. 9, pp. 198-205, 2017.

[27] A. Stefan, I. A. Stanescu, and J. Hauge, "Approaches to Re engineering Digital Games," in ASME. International Design Engineering Technical Conferences and Computers and Infor-mation in Engineering Conference, 2016.

[28] J. Baalsrud Hauge, "An educational framework for supporting the implementation of the Intelligent Cargo con-cept,” Int. J. Adv. Logist., vol. 5, no. 2, pp. 86-100, 2016.

[29] V.-F. F. und -betrieb, Simulation von Logistik-, Materialfluss- und Produktionssystemen. 2014.

[30] K. N. P. Nokelainen, T. Nevalainen, Mind or Machine? Opportunities and Limits of Automation. The Impact of Digitalization in the Workplace. Cham, Switzerland: Springer, 2018.

[31] J. David, A. Lobov, and M. Lanz, "Leveraging Digital Twins for Assisted Learning of Flexible Manufacturing Systems," in IEEE 16th International Conference on Industrial Informatics (INDIN), 2018, pp. 529-535.

[32] D. Jones, C. Snider, A. Nassehi, J. Yon, and B. Hicks, "Characterising the Digital Twin: A systematic literature re view.," CIRP J. Manuf. Sci. Technol., 2020.

[33] E. Negri, L. Fumagalli, and M. Macchi, "A Review of the Roles of Digital Twin in CPS-based Production Systems.," Procedia Manuf., vol. 11, pp. 939-948, 2017.

[34] A. Fuller, Z. Fan, C. Day, and C. Barlo, "Digital Twin: Enabling Technologies, Challenges and Open Research," IEEE Access, vol. 8, pp. 108952-108971, 2020.

[35] M. Holler, F. Uebernickel, and W. Brenner, "Digital Twin Concepts in Manufacturing Industries-A Literature Review and Avenues for Further Research," in Proceedings of 18th International Conference on Industrial Engineering (IJIE). 18th International Conference on Industrial Engineering (IJIE, 2016).

[36] D. Dutta and I. Bose, "Managing a Big Data project: The case of Ramco Cements Limited," Intern. J. Prod. Econ., vol. 165, pp. 293-306, 2015.

[37] P. Lim, Kendrik Yan Hong; Zheng and C.-H. Chen, "A state-of-the-art survey of Digital Twin: techniques, engineering product lifecycle management and business innovation perspectives.,” Jorunal Intell. Manuf., vol. 65, no. $1,2019$. 
[38] E. Barricelli, Barbara Rita; Casiraghi and D. Fogli, "Barricelli, Barbara Rita; Casiraghi, Elena; Fogli, Daniela (2019): A Survey on Digital Twin: Definitions, Characteristics, Applications, and Design Implications.167671.," IEEE Access, vol. 7, p. 167653-167671., 2019.

[39] H. Park, A. Easwaran, and S. Andalam, "Challenges in Digital Twin Development for Cyber-Physical Production Systems.," in Cyber Physical Systems. Model-Based Design, Bd, Roger Chamberlain, W. Taha, and M. Törngren, Eds. Cham, Switzerland: Springer International Publishing, 2019, pp. 28-48.

[40] N. Enders, Martin Robert; Hoßbach, "Dimensions of Digital Twin Applications - A Literature Review.," in In: AMCIS 2019 Proceedings. THE AMERICAS CONFERENCE ON INFORMATION SYSTEMS AMCIS., 2019.

[41] R. B. Roy, D. Mishra, S. K. . Pal, S. Chakravarty, Tapas; Panda, and M. G. et al. Chandra, "Digital twin: current scenario and a case study on a manufacturing process.," Int J Adv Manuf Technol, vol. 107, no. 9-10, pp. 3691-3714, 2020.

[42] T. Y. . Melesse and S. Di Pasquale, Valentina; Riemma, "Digital Twin Models in Industrial Operations: A Systematic Literature Review," Procedia Manuf., vol. 42, pp. 267-272, 2020.

[43] A. Orozco-Romero, C. Y. Arias-Portela, and J. A. M. Saucedo, "The Use of Agent-Based Models Boosted by Digital Twins in the Supply Chain: A Literature Review," in Intelligent Computing and Optimization, Bd., Pandian Vasant, I. Zelinka, and G.-W. W. (Hg.):, Eds. Cham: Spring er International Publishing (Advances in Intelligent Systems and Computing), 2020, pp. 642-652.

[44] L. E. Kavraki, P. Svestka, J. C. Latombe, and M. H. Overmars, "Probabilistic roadmaps for path planning in high-dimensional configuration spaces," IEEE Trans. Robot. Autom., vol. 12, no. 4, pp. 566-580, 1996.

[45] S. M. LaValle and J. J. J. Kuffner, "Randomized kinodynamic planning,” Int. J. Rob. Res., vol. 20, no. 5, pp. 378-400, 2001.

[46] M. Likhachev, "Search-based Planning for Large Dynamic Environments, PhD thesis," Carnegie Mellon University, 2005.

[47] M. Likhachev, G. Gordon, and S. Thrun, "ARA*: Anytime $\mathrm{A}^{*}$ with provable bounds on sub-optimality," in Proceedings of the 16th International Conference on Neural Information Processing, 2003.

[48] P. Mårdberg et al., "AA novel tool for optimization and verification of layout and human logistics in digital factories," Procedia CIRP, 2018.

[49] R. K. Yin, Case Study Research and Applications: Design and Methods, Sixth., vol. 53, no. 9. 2019.

[50] J. Meredith, "Building operations management theory through case and field research,” J. Oper. Manag., vol. Vol.16, no. 4, p. pp.441-454, 1998.

[51] C. Zhuang, J. Liu, and H. Xiong, "Digital twin-based smart production management and control framework for the complex product assembly shop-floor," Int. J. Adv. Manuf. Technol., vol. 96, no. 1-4, pp. 1149-1163, 2018.

[52] HC Pfohl, Produktionslogistik. Berlin: Heidelberg: Springer Berlin Heidelberg, 2018.

[53] J. M. Baalsrud Hauge, A. Engström, I. A. Stefan, and J. Strömgren, "Bridging educational and working environments through pervasive approaches," in 3rd International Joint Conference on Serious Games, JCSG 2017, Springer Verlag , 2017, p. 296-307, 2017, pp. 296-307.

[54] V. Toivonen, M. Lanz, H. Nylund, and H. Nieminen, "The FMS Training Center - a versatile learning environment for engineering education”," Procedia Manuf., vol. Vol. 23, p. 135-140., 2018.

[55] B. Bajic, N. Suzic, N. Simeunovic, S. M. A, and A. Rikalovic, "Real-time Data Analytics Edge Computing Application for Industry 4.0: The Mahalanobis-Taguchi Approach,” Int. J. Ind. Eng. Manag., vol. 11, no. 3, 2020.

[56] E. Hofmann and M. Rüsch, "Industry 4.0 and the current status as well as future prospects on logistics," Comput. Ind., vol. 89, pp. 23-34, 2017. 